\title{
The effects of early rearing conditions upon responses to mirror-image stimulation in domestic chicks and turkeys*
}

\author{
ALLAN H. SCHULMAN and JOHN N. ANDERSON \\ I irginia Polytechnic Institute and State University. Blacksburg. Virginia 24060
}

\begin{abstract}
Two experiments were performed in which either White Plymouth Rock chicks or domestic turkeys were reared under three maintenance conditions (with a mirror, with a conspecific, or in visual isolation) from Day 1 posthatch until Day 7. On Day 7, the Ss were given a preference test to determine whether they spent more time before a mirror or with another bird. Ss reared with a mirror spent approximately two-thirds of their time in the vicinity of the mirror, whereas socially reared Ss spent approximately two-thirds of their time with an agemate. The social isolates, on the other hand, failed to show a preference for either mirror image of conspecific stimulation. A third experiment evaluated the choice behavior of turkeys socially reared in the presence of a mirror, and revealed that, like the social isolates, these Ss also failed to exhibit a reliable preference. The results are interpreted in terms of the effects of early rearing conditions upon social stimulus preferences.
\end{abstract}

Members of numerous vertebrate species, when exposed to their own reflection in a mirror, respond as though they were in the presence of another animal (Gallup. 1968). Birds are particularly responsive to their own reflections (Dickey, 1916; Kaufman \& Hinde, 1961; Smythe. 1962). Evidence is accumulating, however, which suggests that mirror-image stimulation is not identical to normal social stimulation. For example, a decided preference for mirror-image stimulation rather than visual contact with a conspecific has been demonstrated in Siamese fighting fish (Baenninger, 1966), in goldfish (Gallup \& Hess, 1971), and in weaver finches and parakeets (Gallup \& Capper, 1970). Since the observer is the sole determinant of the nature of any encounter with his own reflection, it has been hypothesized by Gallup and Capper (1970) and Gallup and McClure (1971) that mirror-image stimulation may be preferred over normal social contact because of this inherent predictability.

The recent study by Gallup and McClure (1971) revealed this same preference for mirror-image stimulation in surrogate-reared rhesus monkeys but not in feral monkeys. This finding suggests that the conditions of early rearing may be important in determining the attractive potential of mirror-image stimulation. To assess this possibility directly, a series of experiments was performed utilizing domestic chicks and turkeys as Ss. These organisms were chosen because some of their social preferences have been shown to be highly dependent upon their early experiences.

*We wish to express our gratitude to Paul B. Siegel and to A. T. Leighton of the Department of Poultry Science at Virginia Polytechnic Institute and State University for generously providing us with the Ss used in the present study. A preliminary version of this report was read at the annual meeting of the Southeastern Psychological Association, Atlanta, Georgia, 1972.

\section{EXPERIMENT I}

\section{Methods}

Newly hatched White Plymouth Rock chicks (Gallus gallus), incubated in a darkened forced-air incubator, were obtained from the Department of Poultry Science at Virginia Polytechnic Institute and State University and were individually assigned to one of three maintenance conditions: with a mirror $(\mathrm{N}=19)$, with a cagemate $(N=20)$, or in visual isolation $(N=20)$ from Day 1 posthatch until Day 7 . Rearing cages measured $20 \times 20 \times$ $25 \mathrm{~cm}$. Food and water were continuously available. All Ss were maintained at $95^{\circ} \mathrm{F}$ and $50 \%$ relative humidity in an isolation room adjacent to the laboratory. On Day 7 , the Ss were given a single 15 -min preference test to determine whether they spent more time with a mirror or with other chicks.

The testing apparatus consisted of a wooden box measuring $45 \times 30 \times 30 \mathrm{~cm}$ having a hardware cloth floor. A $10 \times 15 \mathrm{~cm}$ mirror was suspended on one end wall of the apparatus. At the opposite end of the apparatus, behind a sheet of plate glass masked to provide a $10 \times 15 \mathrm{~cm}$ opening, were two agemates. Only one of these birds was visible to the S. Two chicks were placed behind the glass because preliminary observations revealed that a single chick often became quite aroused, distress-called, and made persistent attempts to get to the $S$ or to escape from the apparatus. The use of two birds circumvented these problems. Typically, they both remained silent in a resting position. The $\mathrm{S}$ was placed on a red line, $2.5 \mathrm{~cm}$ in width, painted on the floor equidistant between the mirror and the sheet of glass. The amount of time the bird spent on either side of the line was manually determined by using a stopwatch. Orientation of the head was used as an indicator of preference whenever the S stood directly on the boundary line. When a S left the line, it typically proceeded to approach one wall of the apparatus. The position of the stimulus alternatives was varied systematically.

\section{Results}

The average number of seconds the birds in each treatment group spent on either side of the center line of the apparatus is shown in Fig. 1. Open bars represent time spent on the mirror side of the apparatus, while the shaded bars indicate time spent on the conspecific side 


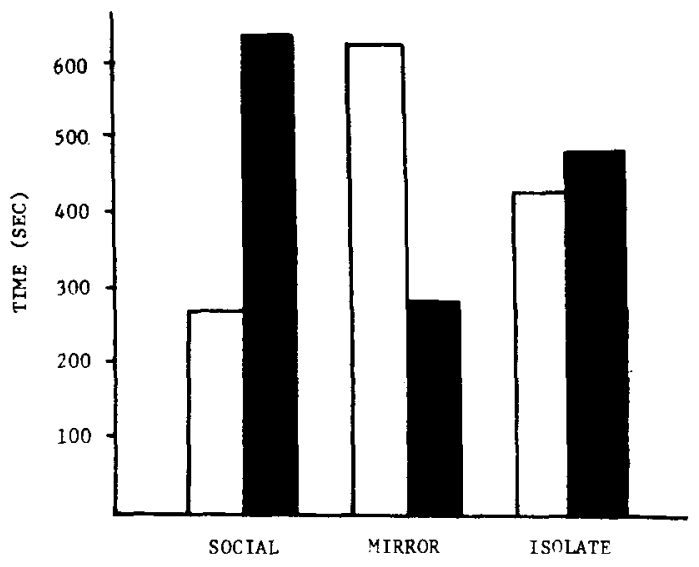

Fig. 1. Mean number of seconds spent on either side of choice apparatus by three groups of chicks. Shaded bars indicate conspecific side of apparatus. Open bars indicate mirror side of apparatus.

of the apparatus. It may be seen that socially reared Ss spent most of their time on the bird-side of the apparatus. Conversely, mirror-reared birds spent most of their time on the mirror side. The isolation-reared birds tended to divide their time equally between both sides of the apparatus.

Because of the nature of the test, the amount of time spent on one side of the dividing line as compared to the other side of the same line are not independent measures. Rather, they are equivalent, since the total trial duration $(900 \mathrm{sec})$ is equal to the sum of the number of seconds spent on either side of the line. Because of this equivalency, an analysis of variance was performed on the amount of time spent on the mirror side of the apparatus. The analysis revealed that differences among the groups were highly significant $(F$ $=22.0, \mathrm{df}=2 / 56, \mathrm{p}<.01)$. Subsequent post hoc analysis with a Scheffé test revealed that each treatment group differed significantly from every other treatment group $(p<.05)$. A binomial test revealed a significant spatial preference for the mirror side of the apparatus in the mirror-reared group $(p=.002)$ and a significant spatial preference for the conspecific side of the apparatus in the socially reared group $(p=.001)$. The isolates failed to show a reliable preference for either alternative $(p=.588)$.

\section{EXPERIMENT II}

The results of Experiment I revealed that domestic chicks show a spatial preference for mirror-image stimulation or for social stimulation with a conspecific, depending upon their conditions of early rearing. This experiment was conducted to determine whether a similar relationship exists for domestic turkeys.

\section{Methods}

Newly hatched domestic turkeys (Meleagris gallopavo) were obtained from the Department of Poultry Science and, as in
Experiment I, were individually assigned to one of three maintenance conditions, i.e., the $S$ s were reared with a mirror $(N$ $=15)$, with conspecifics $(N=15)$, or in visual isolation $(N=17)$ from Day 1 posthatch until Day 7 . Food and water were continuously available. All Ss were maintained at $95^{\circ} \mathrm{F}$ and $50 \%$ relative humidity. On Day 7 , the $S$ s were given a preference test using the apparatus and method described in Experiment I to determine whether they spent more time before a mirror or with other turkeys. The only procedural differences between the present experiment and Experiment I were (1) the 15 social birds were communally reared, in a cage measuring $90 \times 30 \times$ $30 \mathrm{~cm}$, rather than being reared with a single cagemate, and (2) the preference test session was reduced from 15 to $10 \mathrm{~min}$ since a preliminary study revealed that the shorter time interval was sufficient to demonstrate the effect of rearing condition.

\section{Results}

The average number of seconds the turkeys in each treatment group spent on either side of the center line of the choice apparatus is shown in Fig. 2. The results obtained with the turkeys are virtually identical to those obtained with the chicks in Experiment I. Socially reared birds spent most of their time with conspecifics; mirror-reared birds spent most of the test session with their own reflections; the isolates divided their time equally between the two stimulus alternatives. Statistical analyses of the turkey data revealed results identical to those obtained for domestic chicks. An analysis of variance of time spent on the mirror side of the apparatus revealed that the differences among the three groups were highly significant $(\mathrm{F}=31.6, \mathrm{df}=2 / 44$, $\mathrm{p}<.01)$. The Scheffé test showed that each treatment differed significantly from every other treatment group $(p<.05)$. Moreover, a binomial test revealed a reliable preference for mirror-image stimulation in the mirror-reared group $(p<.001)$, and a significant preference for social stimulation in the socially reared group $(\mathrm{p}<.001)$. As was the case with chicks, isolated turkeys failed to show a reliable spatial preference for either their own reflections or for other birds $(p=.500)$.

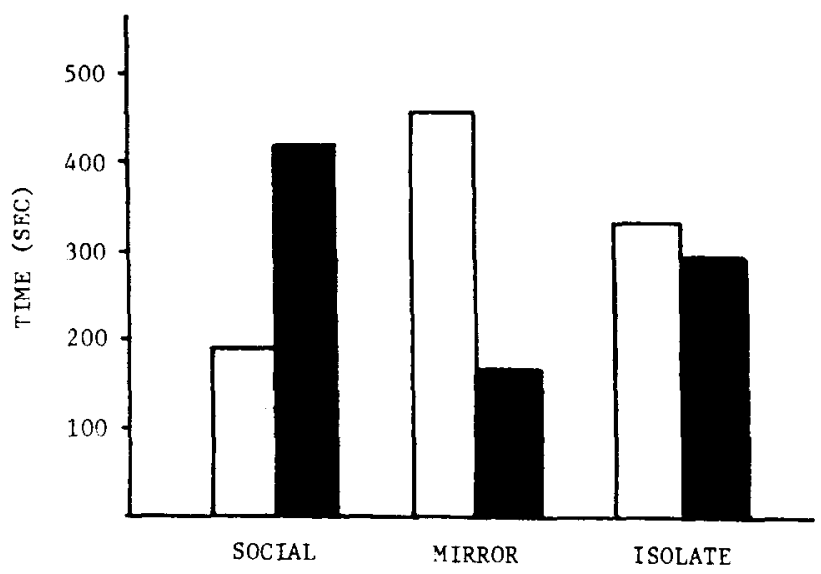

Fig. 2. Mean number of seconds spent on either side of choice apparatus by three groups of turkeys. Shaded bars indicate conspecific side of apparatus. Open bars indicate mirror side of apparatus. 


\section{EXPERIMENT III}

The results of Experiments I and II revealed that in both domestic chicks and turkeys conditions of early rearing affect the birds subsequent spatial preferences for mirror-image stimulation or conspecific stimulation. Birds individually reared in the presence of a mirror prefer mirror-image stimulation. while socially reared birds prefer to remain close to a conspecific. Isolates of both species failed to exhibit a preference. The purpose of the present experiment was to determine the choice behavior of birds raised communally in the presence of a mirror, in order to ascertain whether one aspect of early rearing was more prepotent than the other in influencing the outcome of the preference test.

\section{Method}

Twenty newly hatched turkeys (Meleagris galloparo). obtained from the Department of Poultry Silience. were communally reared in two groups of 10 in cages measuring $60 x$ $30 \times 30 \mathrm{~cm}$ from Day 1 posthatch until Day 7. A mirror measuring $30 \mathrm{~cm}^{2}$ was located on one wall of the rearing cage. Food and water were continuously available. All Si were maintained at $95^{\circ} \mathrm{F}$ and $50 \tau_{c}$ relative humidity. On Day 7 , the Ss were given a 10 -min preference test, using the apparatus and method employed in the two preceding experiments, to determine whether they spent more time with a mirror or with other turkey's.

\section{Results}

Of the 19 turkeys surviving to the test day, 11 animals spent more time on the mirror side of the apparatus. A binomial test revealed that this did not indicate a statistically reliable preference for the mirror $(\mathrm{p}=$ 0.324). Additionally, the mean time spent with the mirror in the 10 -min test was $316.0 \mathrm{sec}$. Consequently, the results of this experiment revealed that when turkeys are reared in the presence of conspecifics and a mirror. they show no preference for either social stimulation of agemates or mirror stimulation when given a choice between the two alternatives.

\section{DISCUSSION}

The results of this study show that in both domestic chicks and turkeys, conditions of early rearing affect the birds' subsequent performance when given a choice between mirror-image stimulation and proximity with a conspecific. Ss raised with mirrors prefer their own reflections; socially reared Ss prefer age mates; isolates do not differentially prefer either mirror-image stimulation or social contact. These results are consistent with the general conclusions of Gallup and McClure
(1971) that differential rearing can affect the preference for mirror-image stimulation. Gallup. Montevecchi and Swanson $(1972)$ found that mirror-image stimulation mas represent a supernormal social stimulus for the elicitation of aggressive responses and the suppression of distress vocalization in a novel situation among socially reared chicts. The results of Experiments I and II suggest that the proposed supernomality of mirror-image stimulation does not include the elicitation of orientational responses of either socially reared chicks or turkeys as measured in the present apparatus. Mureover. the results of Experiment III revealed that when turkeys are reared in the presence of both social and mirror-image stimulation they fail to show a consistent stimulus preference.

Preliminary observations in our laboratory (Schulman \& Anderson. unpublished data) suggest that consistent choice behavior appropriate to rearing condition appears during the 2nd day of postnatal life. Therefore. the reliability of the choice behavior together with the apparent rapidity and accuracy with which such discriminations are established suggest that newly hatched chicks and turkeys are ideally suited for the study of both the effects of early experience on subsequent mirror-image stimulation preferences as well as studies of the prepotent stimulus dimensions of mirtor-image stimulation which underlie such preferences.

\section{REFERENCES}

Baenninger, R. Waning of aggressive motivation in Betta splendens. Psychonomic Science, 1966, 4, 241-242.

Dickey, D. R. The shadow-boxing of Pipilo. The Condor, 1916 $18,93-99$

Gallup, G. G., Jx. Mirror-image stimulation. Psychological Bulletin. $1968,70,782-793$.

Gallup, G. G., Jr., \& Capper, S. A. Preference for mirror-image stimulation in finches (Passer domesticus domesticus) and parakeets (Melopsittacus undulates). Animal Behaviour, 1970 18,621-624.

Gallup, G. G., Jr., \& Hess, J. Y. Preference for mirror-image stimulation in goldfish (Carassius auratus). Psychonomic Science, 1971, 23, 63-64.

Gallup. G. G., Jr., \& McClure, M. K. Preference for mirror-image stimulation in differentially reared rhesus monkeys. Journal of Comparative \& Physiological Psychology, 1971, 75, 403-407.

Gallup, G. G., Jr., Montevecchi, W. A., \& Swanson, E. T. Motivational properties of mirror-image stimulation in the domestic chicken. Psychological Record, 1972, 22, 193-199.

Kaufman, I. C., \& Hinde, R. A. Factors influencing distress calling in chicks with special reference to temperature changes and social isolation. A nimal Behaviour, 1961, 9, 197-204.

Smy the, R. H. Animal habits: The things animals do. Springfield, III: Thomas, 1962.

(Received for publication July 13, 1973; revision received October 26, 1973.) 